IJMMS 29:10 (2002) 585-589

PII. S0161171202007494

http://ijmms.hindawi.com

(c) Hindawi Publishing Corp.

\title{
TRANSLATION INVARIANCE AND FINITE ADDITIVITY IN A PROBABILITY MEASURE ON THE NATURAL NUMBERS
}

\author{
ROBERT GARDNER and ROBERT PRICE
}

\author{
Received 30 April 2001
}

\begin{abstract}
Inspired by the "two envelopes exchange paradox," a finitely additive probability measure $m$ on the natural numbers is introduced. The measure is uniform in the sense that $m(\{i\})=m(\{j\})$ for all $i, j \in \mathbb{N}$. The measure is shown to be translation invariant and has such desirable properties as $m(\{i \in \mathbb{N} \mid i \equiv 0(\bmod 2)\})=1 / 2$. For any $r \in[0,1]$, a set $A$ is constructed such that $m(A)=r$; however, $m$ is not defined on the power set of $\mathbb{N}$. Finally, a resolution to the two envelopes exchange paradox is presented in terms of $m$.
\end{abstract}

2000 Mathematics Subject Classification: 60A10.

1. Introduction. In recent work on the so-called "two envelopes exchange paradox" (cf. Broome [1] and Nalebuff [4]), it has been proposed that the commonly used assumption of countable additivity for probability distributions be dropped [5]. The two envelopes problem is as follows: a random (positive) amount of money is put in an envelope $O$. A coin is flipped and if the coin comes up heads, twice the amount of money in envelope $O$ is put in a second envelope (call it $T$ ) and if the coin comes up tails, half the amount of money in envelope $O$ is put in envelope $T$.

The paradox arises by reasoning that if we choose one envelope (no matter which one) then there is a $50 \%$ chance that the other envelope contains one-half the amount we hold, and there is a 50\% chance that the other envelope contains twice the amount we hold. That is, the other envelope has an expected value of 1.25 times the amount in the envelope we hold. This expected value is greater, regardless of whether we hold envelope $O$ or $T$. Therefore, if we hold envelope $O$ it appears to be to our advantage to swap envelope $O$ for envelope $T$ (we might even be willing to pay a certain amount of money to swap). Also, if we should hold envelope $T$ we also have a desire to swap since we can argue that the expected value in $O$ is 1.25 times the amount in $T$. Of course, the "paradox" is resolved if a probability distribution is given by which the amount of money to be put in envelope $O$ is determined. The expected amount in $O$ is then the expected value of this distribution and the expected amount in $T$ is 1.25 times the expected amount in $O$ [3].

Rawling [5] has suggested addressing the paradox by exploring what happens when the amount in envelope $O$ is based on a natural number $n$ (he chose to put $\$ 2^{n}$ in envelope $O$ ) which is chosen according to a uniform probability distribution on the natural numbers $\mathbb{N}$. With such a distribution, countable additivity of the probability measure must be abandoned. Motivated by this approach, we explore the implications of postulating a uniform probability distribution on $\mathbb{N}$, which satisfies the properties 
of finite additivity and translation invariance. For each real number $r$ such that $0 \leq$ $r \leq 1$, we construct a subset of $\mathbb{N}$, with measure $r$. Finally, we propose a method for calculating expected values for the two envelopes problem which resolves the paradox.

2. The definition and results. We center our discussion around the following definition.

Definition 2.1. Let $S \subset \mathbb{N}$ and $n \in \mathbb{N}$. Then define

$$
c_{S}(n)=|\{x \in S \mid x \leq n\}| .
$$

For $S \subset \mathbb{N}$ define the (probability) measure of $S$ as

$$
P(S)=\lim _{n \rightarrow \infty} \frac{c_{S}(n)}{n},
$$

provided this limit exists.

The probability measure of $S, P(S)$, is occasionally called the asymptotic density of the set $S$ [2]. This definition is also briefly discussed in [6]. Notice that if $A \subset B \subset \mathbb{N}$ then $c_{A}(n) \leq c_{B}(n)$ for all $n \in \mathbb{N}$.

Now, we show that $P$ satisfies several desired conditions for the probability measure of the two envelopes problem.

THEOREM 2.2. Probability measure $P$ satisfies: $P(\varnothing)=0, P(\{n\})=P(\{m\})=0$ for all $m, n \in \mathbb{N}, P(\mathbb{N})=1$, and $P(A)=0$ if $|A|<\infty$.

Proof. This result follows trivially from the definition of $P$.

Theorem 2.2 includes the idea that $P$ should be determined by a uniform distribution. Although finite sets have measure zero, the converse of this result does not hold. Consider, for example, the set

$$
A=\left\{x \mid x=10^{n} \text { for some } n \in \mathbb{N}\right\} .
$$

Then since $c_{A}(n) \leq \log _{10}(n)$, we have $P(A)=0$.

THEOREM 2.3. The probability measure $P$ is finitely additive. That is, if $P\left(S_{1}\right)$, $P\left(S_{2}\right), \ldots, P\left(S_{k}\right)$ are defined, $S_{i} \cap S_{j}=\varnothing$ for $i \neq j$, and $S=S_{1} \cup S_{2} \cup \cdots \cup S_{k}$ is defined, then $P(S)=P\left(S_{1}\right)+P\left(S_{2}\right)+\cdots+P\left(S_{k}\right)$.

Proof. Notice that for a given $n \in \mathbb{N}$,

$$
c_{S}(n)=c_{S_{1}}(n)+c_{S_{2}}(n)+\cdots+c_{S_{k}}(n) .
$$

Therefore,

$$
\begin{aligned}
P(S) & =\lim _{n \rightarrow \infty}\left(\frac{c_{S}(n)}{n}\right)=\lim _{n \rightarrow \infty}\left(\frac{c_{S_{1}}(n)+c_{S_{2}}(n)+\cdots+c_{S_{k}}(n)}{n}\right) \\
& =\lim _{n \rightarrow \infty}\left(\frac{c_{S_{1}}(n)}{n}\right)+\lim _{n \rightarrow \infty}\left(\frac{c_{S_{2}}(n)}{n}\right)+\cdots+\lim _{n \rightarrow \infty}\left(\frac{c_{S_{k}}(n)}{n}\right) \\
& =P\left(S_{1}\right)+P\left(S_{2}\right)+\cdots+P\left(S_{k}\right) .
\end{aligned}
$$


Notice that, in general, $P$ is not countably additive. This follows from the facts that $\mathbb{N}=\bigcup_{n=1}^{\infty}\{n\}, P(\{n\})=0$ for each $n \in \mathbb{N}$, and $P(\mathbb{N})=1$.

We define for $S \subset \mathbb{N}$ and $x \in \mathbb{N}$,

$$
S+x=\{s+x \mid s \in S\}
$$

The set $S+x$ is commonly called a translation for $S$. We have the following theorem.

THEOREM 2.4. The probability measure $P$ is translation invariant. That is, $P(S+$ $x)=P(S)$ for all $S \subset \mathbb{N}$ and for $x \in \mathbb{N}$.

Proof. First, notice that $c_{S}(n) \leq c_{S+x}(n)+x$. Therefore,

$$
\begin{aligned}
P(S) & =\lim _{n \rightarrow \infty} \frac{c_{S}(n)}{n} \leq \lim _{n \rightarrow \infty} \frac{c_{S+x}(n)+x}{n} \\
& =\lim _{n \rightarrow \infty}\left(\frac{c_{S+x}(n)}{n}+\frac{x}{n}\right)=\lim _{n \rightarrow \infty}\left(\frac{c_{S+x}(n)}{n}\right)=P(S+x) .
\end{aligned}
$$

Similarly, since $c_{S}(n) \geq c_{S+x}(n)$, we have $P(S) \geq P(S+x)$. Therefore, $P(S)=P(S+x)$.

3. Sets of given measures. In this section, for any $r \in[0,1]$, we construct a set $A \subset \mathbb{N}$ such that $P(A)=r$. First, we define

$$
A_{i, j}=\{x \in \mathbb{N} \mid x \equiv i(\bmod j)\}
$$

for $i \leq j$ in $\mathbb{N}$.

TheOrem 3.1. Let $p, q \in \mathbb{N}$ with $p \leq q$. Then there exists $A \subset \mathbb{N}$ with $P(A)=p / q$.

Proof. Notice that for all $p, q \in \mathbb{N}, p \leq q$ we have

$$
\frac{n}{q}-1<c_{A_{p / q}}(n)<\frac{n}{q}+1
$$

Therefore, $P\left(A_{p / q}\right)=\lim _{n \rightarrow \infty}\left(c_{A_{p / q}}(n) / n\right)=1 / q$ by the Sandwich theorem. By Theorem 2.3,

$$
P\left(\bigcup_{i=1}^{p} A_{i, q}\right)=\frac{p}{q} .
$$

We now use the decimal expansion of irrational numbers to construct sets of irrational measure.

THEOREM 3.2. For any irrational $r \in[0,1]$, there exists a set $A$ with $P(A)=r$.

Proof. Let the decimal expansion of $r$ be $0 . d_{1} d_{2} d_{3} \cdots$ (i.e., $r=\sum_{i=1}^{\infty} d_{i} \times 10^{-i}$ ). For $k \geq 1$, define

$$
A_{k}=\bigcup_{i=1}^{d_{k}} A_{i \times 10^{k-1} / 10^{k}},
$$


where $A_{i \times 10^{k-1} / 10^{k}}$ is constructed as in Theorem 3.1. Therefore $P\left(A_{k}\right)=d_{k} \times 10^{-k}$. Notice that $A_{i} \cap A_{j}=\varnothing$ if $i \neq j$. Let $A=\bigcup_{i=1}^{\infty} A_{i}$ and let $\epsilon>0$ be given. Then for some $M \in \mathbb{N}, r-\epsilon<0 . d_{1} d_{2} \cdots d_{M}<r$. Let $B_{M}=\bigcup_{i=1}^{M} A_{i}$. Then $B_{M} \subset A$ and $P\left(B_{M}\right)=$ $0 . d_{1} d_{2} \cdots d_{M}$. Now for a given $n, c_{B_{M}}(n) \leq c_{A}(n)$. Therefore, $c_{B_{M}} / n \leq c_{A}(n) / n$ and

$$
r-\epsilon<0 . d_{1} d_{2} \cdots d_{M}=P\left(B_{M}\right)=\lim _{n \rightarrow \infty} \frac{c_{B_{M}}(n)}{n} \leq \liminf _{n \rightarrow \infty} \frac{c_{A}(n)}{n} .
$$

Similarly, there exists $N \in \mathbb{N}$ with

$$
r<0 . d_{1} d_{2} \cdots\left(d_{N}+1\right)<r+\epsilon .
$$

With

$$
B_{N}=\left(\bigcup_{i=1}^{N-1} A_{i}\right) \cup\left(\bigcup_{i=1}^{d_{N}+1} A_{i \times 10^{N-1} / 10^{N}}\right)
$$

we have $A \subset B_{N}$ and $P\left(B_{N}\right)=0 . d_{1} d_{2} \cdots\left(d_{N}+1\right)$. For given $n, c_{A}(n) \leq c_{B_{N}}(n)$ and so $c_{A}(n) / n \leq c_{B_{N}}(n) / n$ and

$$
\limsup _{n \rightarrow \infty} \frac{c_{A}(n)}{n} \leq \lim _{n \rightarrow \infty} \frac{c_{B_{N}}(n)}{n}=0 . d_{1} d_{2} \cdots\left(d_{N}+1\right)<r+\epsilon .
$$

Therefore, for arbitrary $\epsilon>0$ we have

$$
r-\epsilon<\liminf _{n \rightarrow \infty}\left(\frac{c_{A}(n)}{n}\right) \leq \limsup _{n \rightarrow \infty}\left(\frac{c_{A}(n)}{n}\right)<r+\epsilon .
$$

Hence

$$
\liminf _{n \rightarrow \infty}\left(\frac{c_{A}(n)}{n}\right)=\limsup _{n \rightarrow \infty}\left(\frac{c_{A}(n)}{n}\right)=\lim _{n \rightarrow \infty} \frac{c_{A}(n)}{n}=P(A)=r
$$

4. Discussion. Unfortunately, it is fairly easy to construct subsets of $\mathbb{N}$ which are not measurable under our definition. We simply alternate the inclusion and exclusion of larger and larger numbers of natural numbers. For example, define $A_{i}=\left\{10^{2 i-2}+1\right.$, $\left.10^{2 i-2}+2, \ldots, 10^{2 i-1}\right\}$ for $i \in \mathbb{N}$, and define $A=\bigcup_{i=1}^{\infty} A_{i}$. Then for $n=10^{k}$ where $k$ is odd, $c_{A}(n)=9 \times \sum_{i=1}^{(k+1) / 2} 10^{2 i-2}$ and for $n=10^{k}$ where $k$ is even, $c_{A}(n)=9 \times$ $\sum_{i=1}^{k / 2} 10^{2 i-2}$. If we restrict $n$ to values in the set $\left\{n \mid n=10^{k}\right.$ where $k$ is odd $\}$, then $\lim _{n \rightarrow \infty}\left(c_{A}(n) / n\right)=10 / 11$. If we restrict $n$ to values in the set $\left\{n \mid n=10^{k}\right.$ where $k$ is even\}, then $\lim _{n \rightarrow \infty}\left(c_{A}(n) / n\right)=1 / 11$. Therefore $P(A)$ is not defined.

We would now like to return to the two envelopes problem and draw some conclusions from the properties we have developed. We associate a value of $n$ with set $\{n\}$. If we calculate the expected value in envelope $O$ using infinite sums, then we get

$$
\sum_{i=1}^{\infty} i P(\{i\})=\sum_{i=1}^{\infty} 0=0 .
$$

It is not surprising that we get this absurdity when taking an infinite sum, since we have calculated probabilities without having the property of countable additivity. An 
alternative approach is to calculate a "cumulative expected value" and then take a limit. That is, we can argue that the expected amount in envelope $O$ is

$$
\lim _{n \rightarrow \infty}\left(\sum_{i=1}^{n} \frac{C_{\{i\}}(n)}{n} \times i\right)=\lim _{n \rightarrow \infty}\left(\sum_{i=1}^{n} \frac{i}{n}\right)=\lim _{n \rightarrow \infty}\left(\frac{n(n+1)}{2 n}\right)=\infty .
$$

In this way, we calculate a limit of finite sums and never directly deal with an infinite sum. Notice that this gives an infinite expected value for the contents of both envelopes $O$ and $T$, and the paradox is resolved. We therefore propose that, in the setting of the two envelopes problem, probabilities and expected values be computed as above.

\section{REFERENCES}

[1] J. Broome, The two-envelope paradox, Analysis 55 (1995), no. 1, 6-11.

[2] K. L. Chung, A Course in Probability Theory, 2nd ed., Probability and Mathematical Statistics, vol. 21, Academic Press, New York, 1974.

[3] R. Gardner, Commentary on Rawlings, Logic, Probability and Science (N. Shanks and R. Gardner, eds.), Poznan Studies in the Philosophy of the Sciences and the Humanities, vol. 71, Rodopi, Amsterdam, 2000.

[4] B. Nalebuff, The other person's envelope is always greener, Journal of Economic Perspectives 3 (1989), no. 1, 171-181.

[5] P. Rawling, The exchange paradox, finite additivity, and the principle of dominance, Logic, Probability and Science (N. Shanks and R. Gardner, eds.), Poznan Studies in the Philosophy of the Sciences and the Humanities, vol. 71, Rodopi, Amsterdam, 2000.

[6] G. J. Székely, Paradoxes in Probability Theory and Mathematical Statistics, Mathematics and Its Applications (East European Series), vol. 15, D. Reidel Publishing, Dordrecht, 1986.

Robert Gardner: Department of MATHEMATics, Box 70663, EAST TenNessee State UniVERSITY, JOHNSON CITY, TN 37614, USA

E-mail address: gardner r@etsu .edu

Robert Price: Department of MAthematics, Box 70663, EAST TenNESSEe State UniverSITY, JOHNSON CITY, TN 37614, USA

E-mail address: pricejr@etsu.edu 


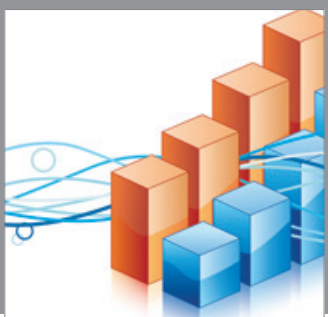

Advances in

Operations Research

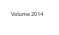

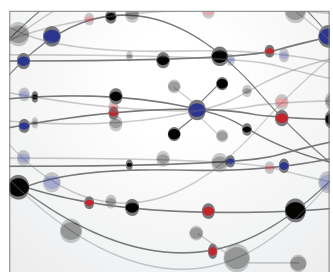

\section{The Scientific} World Journal
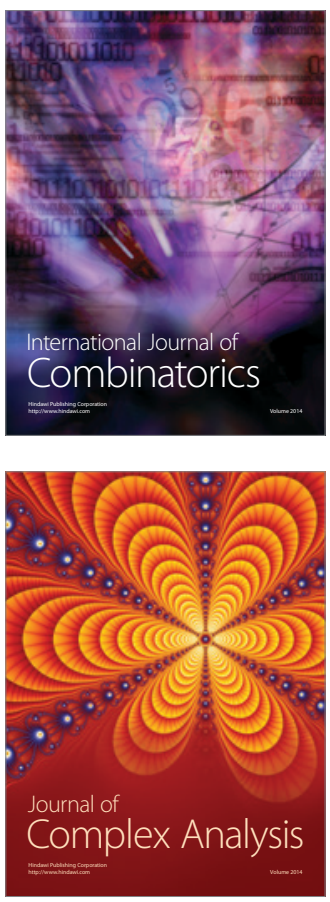

International Journal of

Mathematics and

Mathematical

Sciences
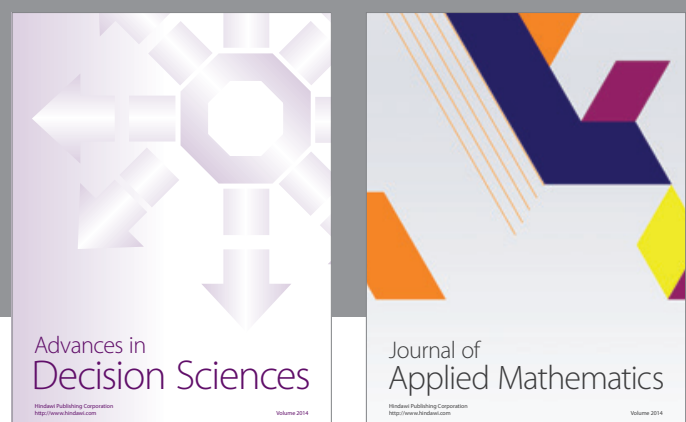

Journal of

Applied Mathematics
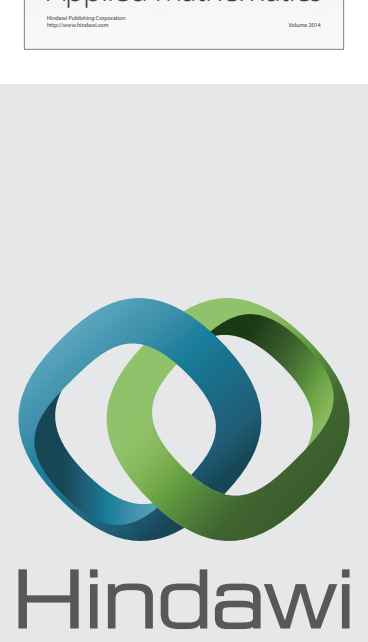

Submit your manuscripts at http://www.hindawi.com
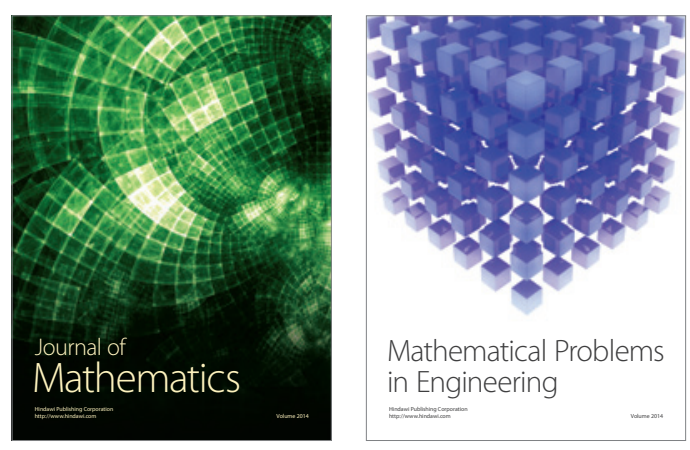

Mathematical Problems in Engineering
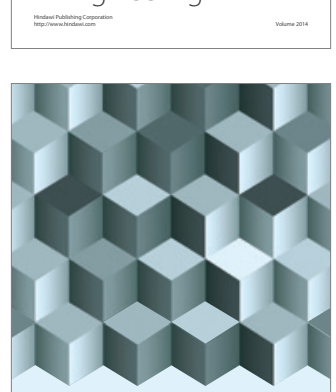

Journal of

Function Spaces
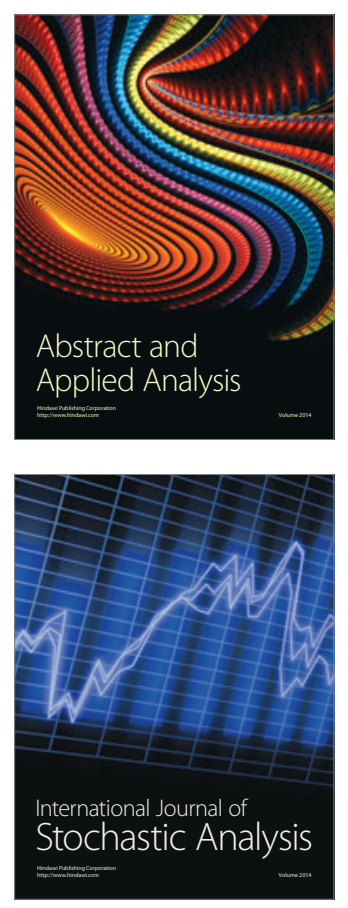

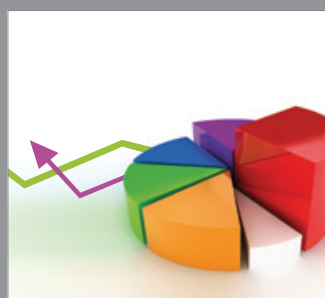

ournal of

Probability and Statistics

Promensencen
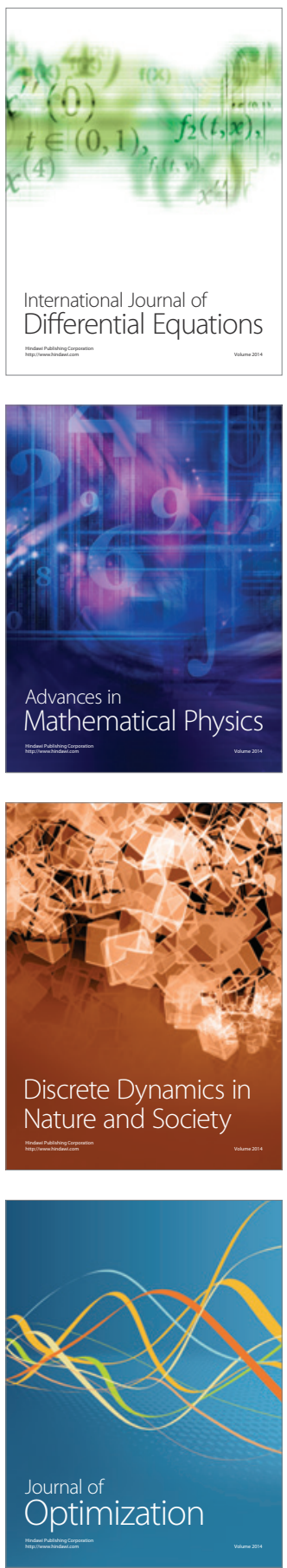